\title{
ON THE SOLVABILITY OF UNIT GROUPS OF GROUP ALGEBRAS
}

\author{
BY \\ J. M. BATEMAN
}

\begin{abstract}
Let $F G$ be the group algebra of a finite group $G$ over a field $F$ of characteristic $p \geqq 0$; and let $U$ be the group of units of $F G$. We prove that $U$ is solvable if and only if (i) every absolutely irreducible representation of $G$ at characteristic $p$ is of degree one or two and (ii) if any such representation is of degree two, then it is definable in $F$ and $F=G F(2)$ or $G F(3)$. This result is translated into intrinsic grouptheoretic and field-theoretic conditions on $G$ and $F$, respectively. Namely, if $O_{p}(G)$ is the maximum normal $p$-subgroup of $G$ and $L=G / O_{p}(G)$, then (i) $L$ is abelian, or (ii) $F=G F(3)$ and $L$ is a 2-group with exactly $\left(|L|-\left[L: L^{\prime}\right]\right) / 4$ normal subgroups of index 8 that do not contain $L^{\prime}$, or (iii) $F=G F(2)$ and $L$ is the extension of an elementary abelian 3-group by an automorphism which inverts every element.

Conditions are found for the nilpotency, supersolvability, and p-solvability of $U$.
\end{abstract}

This paper presents necessary and sufficient conditions for the solvability of the group $U(F G)$ of two-sided units in the group algebra $F G$ of a finite group $G$ over a field $F$. The results are essentially those of the author's doctoral dissertation, presented in a more general form. The author is indebted to D. B. Coleman for many helpful suggestions and for continued encouragement.

In $\S 1$ the contribution of a nilpotent ideal of a ring with 1 to its unit group is considered. Three general results are given in $\$ 2$ for unit groups of finite-dimensional algebras that are separable modulo their radicals. In $\$ 3$ there is given a necessary and sufficient condition for the solvability of the unit group of such an algebra. In $\S 4$ the group $U(F G)$ is considered. Its solvability is related to the representations of $G$ and the nature of $G$ and $F$. The groups $O_{p}(G)$ and $G / O_{p}(G)$ are considered here. In $\S 5$ the solvability, nilpotency, and $p$-solvability of $U(F G)$ are related to the internal structure of $G$.

1. Let $R$ be a ring with 1 and let the set of two-sided units of $R$ be designated $U(R)$.

THEOREM 1. Let $N$ be a nilpotent ideal of $R$. Then the set $1+N$ is a nilpotent normal subgroup of $U(R)$ of class $c \leqq a$, the index of nilpotency of $N$. Furthermore, if $R$ is an algebra over a field $F$ of prime characteristic $p$, then $1+N$ is a p-group with an exponent.

Received by the editors September 14, 1970.

AMS 1969 subject classifications. Primary 2080, 1640; Secondary 2040.

Key words and phrases. Group algebra, unit group, radical, separable algebra, solvability, irreducible representations. 
Proof. That $1+N$ is a normal subgroup is trivial. The nilpotency of $1+N$ and that its class is at most $a$ follows by an easy induction argument on the degree of nilpotency $a$ (cf. [2]).

Suppose further that $R$ is an algebra. Let $p^{e}$ be the least power of $p$ not less than a. Now if $n \in N,(1+n)^{p e}=1+n^{p e}=1$. Thus $1+N$ is a $p$-group and there is an exponent for $1+N$ dividing $p^{e}$.

2. Each finite-dimensional $F$-algebra $A$ has a maximum nilpotent ideal, $\operatorname{rad} A$, called its radical. We have $A / \operatorname{rad} A={ }^{\oplus} \sum_{i=1}^{s} C_{i}$ for simple ideals $C_{i}$, each of which is isomorphic with a total matrix algebra $M_{n_{i}}\left(D_{i}\right)$ of degree $n_{i}$ over a division algebra $D_{i}$ of finite dimension over $F$. We have an obvious

Proposition. $U(A / \operatorname{rad} A) \cong{ }^{\times} \prod U\left(C_{i}\right)$ where $U\left(C_{i}\right) \cong G L\left(n_{i}, D_{i}\right)$.

We assume in this section that $A$ is a finite-dimensional $F$-algebra that is separable modulo its radical; that is, $A / \operatorname{rad} A$ is a separable algebra over $F$. By Wedderburn's Principal Theorem, $A$ has a semisimple subalgebra $S$ with $A / \operatorname{rad} A \cong S$ and $A=S+\operatorname{rad} A$ (vector space direct sum). Clearly $1 \in S$.

For rings with 1 it is easy to see that an epimorphism maps units to units.

LEMMA. Any epimorphism of finite-dimensional semisimple algebras with 1 induces (by restriction) an epimorphism of their unit groups.

Proof. Let $\beta: S_{1} \rightarrow S_{2}$ be such an epimorphism. Let $e$ be the unity for $\operatorname{ker} \beta$ and if $u \in U\left(S_{2}\right)$ let $u^{\prime}$ be its unique pre-image in a complementary ideal for ker $\beta$. Then $\left(e+u^{\prime}\right) \beta=u$ and $e+u^{\prime} \in U\left(S_{1}\right)$.

THEOREM 2. Any epimorphism of finite-dimensional algebras with 1 that are separable modulo their radicals induces an epimorphism of their radicals and an epimorphism of their unit groups.

Proof. Let $\alpha: A_{1} \rightarrow A_{2}$ be the algebra epimorphism. We have $A_{1}=S_{1}+\operatorname{rad} A_{1}$ for semisimple $S_{1}$. Since $1 \in S_{1}, U\left(S_{1}\right) \leqq U\left(A_{1}\right)$. Hence $A_{2}=\alpha\left(S_{1}\right)+\alpha\left(\operatorname{rad} A_{1}\right)$, a sum of a semisimple subalgebra and a nilpotent ideal. Since $1=\alpha(1) \in \alpha\left(S_{1}\right)$, $U\left(\alpha\left(S_{1}\right)\right) \leqq U\left(\alpha\left(A_{1}\right)\right)=U\left(A_{2}\right)$. Clearly $\alpha\left(\operatorname{rad} A_{1}\right) \cap \alpha\left(S_{1}\right)=0$ since it is a nilpotent ideal of $\alpha\left(S_{1}\right)$; consequently, $A_{2}=\alpha\left(S_{1}\right)+\alpha\left(\operatorname{rad} A_{1}\right)$, a vector space direct sum and there is an epimorphism $\beta$ of $A_{2}$ onto $\alpha\left(S_{1}\right)$ defined by

$$
\beta(s+r)=s \text { for } s \in \alpha\left(S_{1}\right) \text { and } r \in \alpha\left(\operatorname{rad} A_{1}\right) \text {. }
$$

Since $A_{2} / \alpha\left(\operatorname{rad} A_{1}\right) \cong \alpha\left(S_{1}\right), \alpha\left(\operatorname{rad} A_{1}\right)=\operatorname{rad} A_{2}$.

Let $u_{2} \in U\left(A_{2}\right)$. Then $u_{2}=s_{2}+r_{2}, s_{2} \in \alpha\left(S_{1}\right), r_{2} \in \operatorname{rad} A_{2}$. The unit $s_{2}=\beta\left(u_{2}\right)$ of $\alpha\left(S_{1}\right)$ is $\alpha\left(s_{1}\right)$ for some $s_{1} \in U\left(S_{1}\right)$ by the lemma; $r_{2}$ is the image of some $r_{1} \in \operatorname{rad} A_{1}$. Now $u_{1}=s_{1}+r_{1}$ is a unit of $A_{1}$ with inverse $\left(1+s_{1}^{-1} r_{1}\right)^{-1} s_{1}^{-1}$ and we have $\alpha\left(u_{1}\right)$ $=\alpha\left(s_{1}\right)+\alpha\left(r_{1}\right)=u_{2}$.

THEOREM 3. Let $A$ be a finite-dimensional algebra with 1 that is separable modulo its radical. Then $U(A)$ is a split extension of $1+\operatorname{rad} A$ by $U(A / \operatorname{rad} A)$. 
Proof. We can assume $A=S+\operatorname{rad} A$. As in the last proof there is an epimorphism $\beta: A \rightarrow S$ with $\beta(s+r)=s$. Hence $S \cong A / \operatorname{rad} A$ and $U(S) \cong U(A / \operatorname{rad} A)$. If $s \in U(S)$ and $r \in \operatorname{rad} A$, then $(s+r)^{-1}=\left(1+s^{-1} r\right)^{-1} s^{-1}$. Therefore the restriction $\beta_{u}=\beta \mid U(A)$ is an epimorphism of $U(A)$ onto $U(S)$ with $\operatorname{ker} \beta_{u}=1+\operatorname{rad} A$. Finally, $U(S) \leqq U(A)$ and the inclusion map is a splitting isomorphism for $\beta_{u}$.

Next we characterize $1+\operatorname{rad} A$. Define $O_{p}(H)$ to be the maximum normal $p$ subgroup of a group $H$.

Lemma. $O_{p}(G L(n, D))=1$ if $D$ is a division ring of characteristic $p$.

Proof. Let $P$ be a normal $p$-subgroup of $G L(n, D)$. For $a \in D, a^{p}=1$ only if $a=1$; hence $O_{p}(G L(1, D))=1$. Next, $O_{p}(G L(2, D))=1$ for $D=G F(2)$ or $G F(3)$, by direct computation. Finally, suppose $P \neq 1$ and either $n \geqq 3$ or both $n=2$ and $|D| \geqq 4$. By Theorem 4.9 of [1], $P \leqq$ Center $(G L(n, D))$ or $S L(n, D) \leqq P$. In the first case there is a nonsingular $\pi$ of order $p$ and an $a \in Z^{*}=\operatorname{Center}(D)-\{0\}$ for which $\pi(v)=v a$ for all vectors $v$. Hence $v a^{p}=v, a^{p}=1, a=1$, and $\pi=1$, a contradiction. On the other hand, since $\left(\begin{array}{cc}0 & 1 \\ -1 & 1\end{array}\right) \in S L(n, D)$ and has order 3 or 6 for $p=2$ or $p>2$, respectively, we cannot have $S L(n, D) \leqq P$. Hence $P=1$ and the lemma is proved.

THeOREM 4. $1+\operatorname{rad} A=O_{p}(U(A))$ if $A$ is a finite-dimensional F-algebra with 1 that is separable modulo its radical and $F$ has characteristic $p$, a prime.

Proof. We have $U(A) / 1+\operatorname{rad} A \cong \prod_{i} G L\left(n_{i}, D_{i}\right)$ and so $O_{p}(U(A) / 1+\operatorname{rad} A)$ $\cong{ }^{\times} \prod_{i} O_{p}\left(G L\left(n_{i}, D_{i}\right)\right)$. But $1+\operatorname{rad} A \leqq O_{p}(U(A))$. Hence $1+\operatorname{rad} A=O_{p}(U(A))$.

3. We maintain the assumptions of $\S 2$ on the algebra $A$. If $U(A)$ is solvable, then its homomorph $U(A / \operatorname{rad} A) \cong{ }^{\times} \prod_{i=1}^{s} G L\left(n_{i}, D_{i}\right)$ and hence also the groups $G L\left(n_{i}, D_{i}\right)$ are all solvable.

Suppose $n>1$ for $H=G L(n, D)$. If $D$ is an infinite field, $H$ is not solvable; if $D$ is a finite division ring then $H$ is solvable if and only if $n=2$ and $D=G F(2)$ or $G F(3)$ [9]. If $D$ is infinite and not commutative, then $U(D)$ is not solvable [8] and for any $n, H \geqq U(D)$ and is not solvable.

We have a

Proposition. If $D$ is a division ring, $G L(n, D)$ is solvable if and only if

(a) $n=1$ and $D$ is a field, or

(b) $n=2$ and $D=G F(2)$ or $G F(3)$.

We can now prove our first solvability theorem.

THEOREM 5. If $A$ is an algebra over $F$ with 1 that is finite dimensional and separable modulo its radical, then $U(A)$ is solvable if and only if in the decomposition

$$
A / \operatorname{rad} A \cong \sum_{i=1}^{s} M_{n_{i}}\left(D_{i}\right)
$$

either 
(1) each $n_{i}=1$ and each $D_{i}$ is a field, or

(2) each $n_{i}=1$ or $2, F=G F(2)$ or $G F(3), D_{\imath} \cong F$ whenever $n_{i}=2$, and some $n_{i}=2$.

Proof. From the above it is clear that for $U(A)$ to be solvable it is necessary that each $n_{i}=1$ and each $U\left(D_{i}\right)$ be solvable or that (2) hold.

Notice that in condition (2) each $D_{i}$ is finite and hence a field.

The alternatives (1) and (2) are sufficient since in either case the groups $G L\left(n_{i}, D_{i}\right)$ are all solvable and $U(A)$ is an extension by their direct product of the group $1+\operatorname{rad} A$.

In preparation for Theorem 6 we establish a

Lemma. Let $F^{*}$ be the algebraic closure of $F$. For an F-algebra separable modulo its radical, condition (2) of Theorem 5 holds if and only if

(i) every irreducible representation of $A \otimes F^{*}$ is of degree one or two and

(ii) some irreducible representation is of degree two and any such representation is definable over $F=G F(2)$ or $G F(3)$ (i.e., it is afforded by $M \otimes F^{*}$ for some irreducible left A-module $M$ ).

Proof. Assume condition (2) of Theorem 5. Then $A / \mathrm{rad} A=\sum_{i \in I_{1} \cup I_{2}} B_{i}, B_{i}$ $\cong M_{2}(F)$ for $i \in I_{2}, B_{i} \cong G F\left(q_{i}\right)$ for $i \in I_{1}, I_{1} \cap I_{2}=\varnothing, F=G F(p)$, and $p=2$ or 3 .

Let $S$ be one of the subalgebras of $A$ isomorphic with $A / \operatorname{rad} A$. Then $S \otimes_{F} F^{*}$ is semisimple over $F^{*}$. Furthermore, $\operatorname{rad} A \otimes_{F} F^{*}$ is a nilpotent ideal of $A \otimes_{F} F^{*}$. Since $A=S+\operatorname{rad} A$ as an $F$-space, $A \otimes_{F} F^{*}=\left(S \otimes_{F} F^{*}\right) \dot{+}\left((\operatorname{rad} A) \otimes_{F} F^{*}\right)$ as an $F^{*}$-space. Thus $\operatorname{rad}\left(A \otimes_{F} F^{*}\right)=(\operatorname{rad} A) \otimes_{F} F^{*}$ and $\left(A \otimes F^{*}\right) / \operatorname{rad}\left(A \otimes F^{*}\right)$ $\cong(A / \operatorname{rad} A) \otimes F^{*}$.

The algebra $\left(A \otimes F^{*}\right) / \operatorname{rad}\left(A \otimes F^{*}\right)$ decomposes as

$$
\sum_{j \in J} C_{j} \cong \sum_{i \in I_{1} \cup I_{2}} B_{i} \otimes F^{*}
$$

with $C_{j} \cong M_{m}\left(D_{j}^{*}\right)$ and $D_{j}^{*}$ a division algebra of finite dimension over $F^{*}$. It follows that each $D_{j}^{*} \cong F^{*}$. The isomorphism $\left(^{*}\right)$ induces a partition of $J$ into sets $J_{1}$ and $J_{2}$ and induces isomorphisms

$$
\sum_{j \in J_{k}} C_{j} \cong \sum_{i \in I_{k}} B_{i} \otimes F^{*} \text { for } k=1,2 .
$$

Since $\sum_{i \in I_{1}} B_{i} \otimes F^{*}$ is commutative, $m_{j}=1$ for $j \in J_{1}$. For $i \in I_{2}, B_{i} \otimes F^{*} \cong M_{2}\left(F^{*}\right)$, which is simple; and so $B_{i} \otimes F^{*}$ must correspond to one $C_{j}$ with $j \in J_{2}$. This results in a one-to-one correspondence $\alpha$ of $I_{2}$ onto $J_{2}$. It also shows that $m_{j}=2$ if $j \in J_{2}$. Thus $\left(A \otimes F^{*}\right) / \operatorname{rad}\left(A \otimes F^{*}\right) \cong \sum_{j \in J_{1}} M_{1}\left(F^{*}\right) \oplus \sum_{i \in I_{2}} M_{2}\left(F^{*}\right)$ and the irreducible representations of $A \otimes F^{*}$ are of degrees one and two [5, p. 171]. Since $I_{2} \neq \varnothing$, some of these are of degree two.

Let $R$ be an irreducible representation of $A \otimes F^{*}$ of degree two, choosing as representation space any minimal left ideal $L^{*}$ of $C_{j} \cong M_{2}\left(F^{*}\right)$. Then $j \in J_{2}$ and it corresponds under $\alpha$ to some $i \in I_{2}$. Let $L$ be a minimal left ideal of $B_{i}$. Then $B_{i} \cong M_{2}(F)$ and $L \otimes F^{*}$, regarded naturally as a subspace of $B_{i} \otimes F^{*}$, is a minimal 
left ideal of $B_{i} \otimes F^{*} \cong C_{j}$. Hence $L \otimes F^{*} \cong L^{*}$ as a representation space and the left $A$-module $L$ affords an irreducible representation of $A$ proving that $R$ is definable over $F$.

For the converse assume (i) and (ii) of the lemma so that $\left(A \otimes F^{*}\right) / \operatorname{rad}\left(A \otimes F^{*}\right)$ $=\sum C_{j}$ for simple $C_{j}, C_{j} \cong M_{m_{j}}\left(F^{*}\right)$ with $m_{j}=1$ or 2 , and at least one $m_{j}=2$. Similarly, $A / \operatorname{rad} A=\sum B_{i}$ with $B_{i} \cong M_{n_{i}}\left(D_{i}\right)$ for some finite (commutative) division algebra $D_{i}$ over $F=G F(2)$ or $G F(3)$.

There is an isomorphism $\eta: \sum C_{j} \rightarrow \sum B_{i} \otimes F^{*}$ so for each $C_{j} \cong M_{2}\left(F^{*}\right)$ there is a $B_{i}$ such that the $\eta$-pre-image of $B_{i} \otimes F^{*}$ contains $C_{j}$, i.e., $D \oplus C_{j} \cong B_{i} \otimes F^{*}$ where $D$ is a sum of $C_{k}$ 's. Let $L$ be a minimal left ideal of $B_{i}$; then $L \otimes F^{*}$ is a representation module for $A \otimes F^{*}$ and is a direct sum containing at least one copy of each of the irreducible modules afforded by simple components of $B_{i} \otimes F^{*}$, since the regular representation of $(A / \operatorname{rad} A) \otimes F^{*}$ is equivalent to the scalar extension of the regular representation of $A / \operatorname{rad} A$. Hence any minimal left ideal $L^{*}$ of $C_{j}$ is isomorphic with some summand of $L \otimes F^{*}$. However, by assumption, $L^{*} \cong M \otimes F^{*}$ for some irreducible module $M$ of $A$. Thus $L \otimes F^{*}$ and $M \otimes F^{*}$ have a common composition factor and hence so do $L$ and $M$ [5, p. 200], i.e., $L \cong M$ and $L^{*} \cong L \otimes F^{*}$.

Hence $B_{i} \otimes F^{*}$ has only the one component $\eta\left(C_{j}\right)$ which means $M_{n_{i}}\left(D_{i}\right) \otimes F^{*}$ $\cong M_{2}\left(F^{*}\right)$ and, since now $n_{i} \operatorname{dim}_{F^{*}} D_{\iota}=2, n_{i}=2$ and $D_{i} \cong F$. Thus for each component $C_{j}$ with $m_{j}=2, \eta\left(C_{j}\right)=B_{i} \otimes F^{*}$ with $n_{i}=2$ and $D_{i} \cong F$. If $C$ is the sum of the commutative $C_{j}$ 's, then $\eta(C)=B \otimes F^{*}$ where $B$ is the sum of the remaining $B_{i}$ 's. Thus each of these $B_{i}$ 's is of the form $M_{1}\left(D_{i}\right)$, i.e., $n_{i}=1$ if $n_{i} \neq 2$ or $D_{i} \not F$.

4. The motivation for the last two sections is the fact that if $F$ is a field and $G$ a finite group then $F G$ is separable modulo its radical. Thus $U(F G)$ is a split extension of $1+\operatorname{rad} F G$ by a direct product ${ }^{\times} \prod_{i} U\left(C_{i}\right)$ where $C_{i} \cong M_{n_{i}}\left(D_{i}\right)$ and $U\left(C_{i}\right)$ $\cong G L\left(n_{i}, D_{i}\right)$.

The radical is nontrivial only when $F$ has prime characteristic $p$ in which case $1+\operatorname{rad} F G$ is the maximum normal $p$-subgroup of $U(F G)$ and has an exponent. Any group epimorphism $\eta: G \rightarrow H$ extends linearly to an algebra epimorphism $\eta^{\prime}: F G \rightarrow F H$; hence, the restriction $\eta_{u}^{\prime}: U(F G) \rightarrow U(F H)$ is an epimorphism.

THEOREM 6. Let $G$ be a finite group and $F$ be a field of characteristic $p \geqq 0$. Then $U(F G)$ is solvable if and only if

(i) every absolutely irreducible representation of $G$ at characteristic $p$ is of degree one or two and

(ii) if any such representation is of degree two, then it is definable in $F$ and $F=G F(2)$ or $G F(3)$.

Proof. By Theorem 5 and the lemma of $\S 3$ we know $U(F G)$ is solvable if and only if one of the two conditions below occurs. Let $A=F G$ and let $F^{*}$ be the algebraic closure of $F$. 
Condition 1. $A / \operatorname{rad} A \cong \sum_{i=1}^{s} D_{i}$ with each $D_{i}$ a field. This is equivalent to having all absolutely irreducible representations $\bar{\rho}_{i}$ of $G$ of degree one. If each $D_{i}$ is commutative then each $\bar{\rho}_{i}$ has degree one. If each $\bar{\rho}_{i}$ has degree one, then $(A / \operatorname{rad} A) \otimes F^{*}$ is a sum of fields; hence, so is $A / \operatorname{rad} A$ and Condition 1 holds.

Condition 2. Every irreducible representation of $A \otimes F^{*}$ is of degree one or two, some irreducible representation is of degree two, and any of degree two is definable over $F$, which must by $G F(2)$ or $G F(3)$.

Since there is a one-to-one degree-preserving correspondence between the irreducible representations of $A \otimes F^{*}$ and the absolutely irreducible representations of $G$ over $F^{*}$, this condition is equivalent to the conjunction of (i) and (ii) of the theorem with the condition that there be at least one absolutely irreducible representation of degree two for $G$.

THEOREM 7. If $G$ is a finite group and $F$ is a field of characteristic $p$, a prime, and $\rho_{1}, \ldots, \rho_{s}$ is a complete set of inequivalent absolutely irreducible representations of $G$ at characteristic $p$ then

(i) $O_{p}(G)=G \cap(1+\operatorname{rad} F G)$ and

(ii) $G / O_{p}(G)$ is a subdirect product of $\rho_{1}(G), \ldots, \rho_{s}(G)$.

Proof. (i) Let $P$ be a normal $p$-subgroup of $G$. Then $[6$, p. 36] the left ideal of $F G$ generated by the elements $g-1, g \in P$, is a nilpotent two-sided ideal of $F G$ and, consequently, is a subset of $\operatorname{rad} F G$ so that $P-1 \subseteq \operatorname{rad} F G$. Hence $O_{p}(G)$ $\subseteq G \cap(1+\operatorname{rad} F G)$. By Theorem $1, G \cap(1+\operatorname{rad} F G)$ is a normal $p$-subgroup and must be contained in $O_{p}(G)$.

(ii) Consider the $\rho_{i}$ 's as matrix representations and define

$$
\rho(g)=\rho_{1}(g) \oplus \cdots \oplus \rho_{s}(g),
$$

the direct sum of these matrices. Then $\rho$ is a matrix representation of $G$ by a subdirect product of $\rho_{1}(G), \ldots, \rho_{s}(G)$. Finally, $\rho(g)=1$ if and only if each $\rho_{i}(g)=1$, which happens precisely when $g \in G \cap(1+\operatorname{rad} F G)=O_{p}(G)$.

5. In this section is given a group-theoretic characterization of finite groups $G$ for which $U(F G)$ is solvable. Theorems 8,10 , and 11 contain the characterization in view of Theorem 6 . Theorems 13, 14 and 15 take up nilpotence and $p$-solvability.

THEOREM 8. The first three conditions below are equivalent and they imply the fourth.

(i) $G^{\prime}$ is a p-group.

(ii) $G / O_{p}(G)$ is abelian.

(iii) All absolutely irreducible representations of $G$ at characteristic $p$ are linear.

(iv) $U(F G)$ is solvable.

Proof. By Theorem 6, (iii) implies (iv). Conditions (i) and (ii) are clearly equivalent. From Theorem 7, (ii) holds if and only if each absolutely irreducible $\rho_{i}(G)$ is abelian and hence of degree one (linear). 
It follows from this theorem that if $H$ is a group of matrices over $F$ of degree $n>1$ and is an abelian extension of a $p$-group $(p=$ char $F)$ then $H$ is not absolutely irreducible. We will also need the following fact in later arguments: If $A \leqq G L(2, p)$ and $A$ is reducible over some field of characteristic $p$, then $A$ is abelian or $p|| A \mid$. To see this consider the map

$$
\left(\begin{array}{ll}
a & 0 \\
c & b
\end{array}\right) \rightarrow\left(\begin{array}{ll}
a & 0 \\
0 & b
\end{array}\right)
$$

We will have use for the following lemma.

LEMMA 1. If $U(F S)$ is solvable, $|S|=n$, S has $s$ inequivalent absolutely irreducible representations $\rho_{i}$ at characteristic $p, l$ of these are of degree one, and $p \nmid n$, then

(i) $n=l+4(s-l)$,

(ii) $l=\left[S: S^{\prime}\right]$, and

(iii) $s-l$ is the number of $\rho_{i}$ 's of degree two.

Proof. Immediate from Theorem 6 and [5].

Suppose $U(F G)$ is solvable and $G^{\prime}$ is not a $p$-group. Then by Theorems 6 and 8 , $F=G F(p), p=2$ or 3 and for each nonlinear absolutely irreducible representation $\rho_{i}$ of $G$ at characteristic $p, \rho_{i}$ is definable over $F$. By Theorem 7 and the remark about epimorphisms at the beginning of $\S 4$, each $U\left(F \rho_{i}(G)\right)$ is solvable. Therefore, in the subdirect factorization of $G / O_{p}(G)$, the groups $\rho_{i}(G)$ that are nonabelian can be thought of as absolutely irreducible subgroups $S$ of $G L(2, F)$ with the added property that $U(F S)$ is solvable.

Suppose $S$ is such a group. Clearly $S^{\prime}$ is not a $p$-group by the remarks following Theorem 8.

For $p=2, G L(2, F) \cong D_{3}$. But $G L(2, F)$ is absolutely irreducible and $U\left(F D_{3}\right)$ $\cong C_{2} \times D_{3}$, where $C_{m}$ will mean henceforth a cyclic group of order $m$. Hence $S=G L(2, F) \cong D_{3}$.

For $p=3$, we need only to consider $S$ when $|S| \in\{8,12,16,24,48\}$ since $|G L(2, F)|=48$. First we examine two groups of order eight. The quaternion group $Q$ has four linear representations and the faithful irreducible representation. By [5] these are the absolutely irreducible representations and by Theorem $6 U(F Q)$ is solvable. Similarly the dihedral group $D_{4}$ has four linear representations and the faithful irreducible one, these are the absolutely irreducible representations, and $U\left(F D_{4}\right)$ is solvable.

If $|S|=8, S \cong Q$ or $D_{4}$; the faithful representation is unique up to equivalence. Hence $S$ is equivalent (conjugate) to

$$
Q=\left\langle\left(\begin{array}{ll}
1 & 1 \\
1 & 2
\end{array}\right),\left(\begin{array}{ll}
0 & 1 \\
2 & 0
\end{array}\right)\right\rangle \quad \text { or } \quad D_{4}=\left\langle\left(\begin{array}{ll}
2 & 2 \\
0 & 1
\end{array}\right),\left(\begin{array}{ll}
1 & 0 \\
1 & 2
\end{array}\right)\right\rangle
$$

If $|S|=12, S$ is dihedral since any subgroup of order 12 of $G L(2,3)$ is dihedral. Consequently, $S^{\prime}$ is a $p$-group, a contradiction. 
If $|S|=16, U(F S)$ is not solvable. To see this, notice first that $S$ is conjugate to

$$
\Sigma=\left\langle A=\left(\begin{array}{ll}
0 & 1 \\
1 & 1
\end{array}\right), B=\left(\begin{array}{ll}
2 & 1 \\
1 & 1
\end{array}\right)\right\rangle .
$$

Since $(A, B)=A^{2},\left|\Sigma^{\prime}\right|=\left[\Sigma: \Sigma^{\prime}\right]=4$.

Since $U(F S)$ is solvable, $S$ must have, according to the lemma after Theorem 8 , $(16-4) / 4=3$ absolutely irreducible representations of degree two definable in $F$. The kernel of such a representation must be $\langle I\rangle$ or $\langle-I\rangle$; there are, up to equivalence, only two such representations, again a contradiction. In particular, $U(F \Sigma)$ is not solvable.

If $|S|=48, U(F \Sigma) \leqq U(F S)$ so $U(F S)$ is not solvable.

Suppose $|S|=24$. Write $L$ for $G L(2,3)$. Then $L^{\prime}=S$, the unique subgroup of $L$ of order 24. Consider any absolutely irreducible representation of $L^{\prime}$ in $L$ with kernel (say) $N$. If 3||$N \mid$, then $N$ contains all 3 -elements, and $|N| \geqq 12$, but then $\left|L^{\prime}\right| N \mid \leqq 2$ and the representation is not absolutely irreducible after all. Suppose then that $N$ is a 2-group. Then $N \leqq Q=L^{\prime \prime}$. The subgroups of order 4 of $L^{\prime}$ are not normal in $L^{\prime}$; hence $N=\langle I\rangle,\langle-I\rangle$, or $Q$ and $\left|L^{\prime}\right| N \mid=24,12$, or 3, respectively. Unless $N=\langle I\rangle$, the representation is not absolutely irreducible and so $L^{\prime}$ has at most one absolutely irreducible representation in $L$. But $L^{\prime}$ has only one linear representation and it has three 3-regular classes, so $U(F S)$ is not solvable [3].

The proof of the following lemma is now complete.

LEMMA 2. If $S \leqq G L(2, p)$ and $p=2$ or 3 then $S$ is absolutely irreducible and $U(G F(p) S)$ is solvable if and only if $p=2$ and $S=G L(2,2) \cong D_{3}$ or $p=3$ and $S$ is quaternion or dihedral of order 8.

Lemma 3. Suppose $F$ has prime characteristic $p, A \times G$ is finite, $U(F G)$ is solvable, $A$ is abelian of order relatively prime to $p$, and $G^{\prime}$ is not a $p$-group. Then $U(F(A \times G))$ is solvable if and only if

(i) $F=G F(2)$ and $A=1$ or

(ii) $F=G F(3)$ and $A$ has exponent 2.

Proof. Clearly $U(F(A \times G))$ is solvable, under the assumptions stated, if (i) holds. If (ii) holds then $A \cong{ }^{\times} \prod C_{2}, F A \cong{ }^{\otimes}\left\lceil C_{2} \cong \sum F, F(A \times G) \cong F A \otimes_{F} F G\right.$ $\cong\left(\sum F\right) \otimes_{F} F G \cong \sum F G$, and finally, $U(F(A \times G)) \cong{ }^{\times} \prod U(F G)$ which is solvable.

Assume $U(F(A \times G))$ is solvable. Since $A$ has order prime to $p$ and is commutative, $F A \cong \sum F_{i}$ for fields $F_{i}$ over $F$. Thus $F(A \times G) \cong F A \otimes_{F} F G \cong \sum F_{i} \otimes_{F} F G$ $\cong \sum F_{i} G$. The solvability of $U(F(A \times G))$ now implies the solvability of each group $U\left(F_{i} G\right)$. Since $G$ is not an abelian extension of a $p$-group, each field $F_{i} \cong G F(2)$ or $G F(3)$. If $p=2$, then $F A \cong \sum G F(2), U(F A)=1, A=1$. If $p=3, F A \cong \sum G F(3)$, $U(F A) \cong{ }^{\times} \prod C_{2}, A^{2}=1$.

For reference, here are some facts on subdirect products. See [7, p. 63]. Let $S \leqq G \times H, M=\{g \in G \mid(g, 1) \in S\}, N=\{h \in H \mid(1, h) \in S\}$. Then $S$ is a subdirect 
product of $G$ and $H$ if the projections of $S$ into $G$ and $H$ are onto. If $S$ is a subdirect product,

(i) there is an isomorphism $m: G / M \rightarrow H / N$ such that $(g, h) \in S$ if and only if $m(g M)=h N$;

(ii) $M \times N$ is the maximal external direct product contained in $S$ and will be designated cart $S$;

(iii) if $\alpha$ and $\beta$ are homomorphisms on $G$ and $H$, respectively, with kernel $\alpha \leqq M$ and kernel $\beta \leqq N$, then $S^{*}=\{(\alpha(g), \beta(h)) \mid(g, h) \in S\}$ is a subdirect product of $\beta(G)$ and $\beta(H)$ with cart $S^{*}=\alpha(M) \times \beta(N)$, and $\alpha(G) / \alpha(M) \cong \beta(H) / \beta(N) \cong G / M \cong H / N$;

(iv) if $N=1, S \cong G$;

(v) if $M=G$ and $N=H$, then $S=G \times H$;

(vi) if $S$ is a subdirect product of $G_{1}, \ldots, G_{n}$ with elements written $\left(g_{1}, g_{2}, \ldots, g_{n}\right), g_{i} \in G_{i}$, then each mapping $\left(g_{1}, g_{2}, \ldots, g_{n}\right) \rightarrow\left(g_{i_{1}}, g_{i_{2}}, \ldots, g_{i_{k}}\right)$ is a homomorphism onto a subdirect product of $G_{i_{1}}, \ldots, G_{i_{k}}$.

In particular, if in (vi) for each distinct pair $i_{1}<i_{2}$ the resulting product of $G_{i_{1}}$ and $G_{i_{2}}$ is not a direct product we will say that $S$ is pairwise nondirect.

If (i) holds for normal $M$ and $N$, then $S$ is a subdirect product of $G$ and $H$.

THEOREM 9. The following three conditions are equivalent.

(i) The unit group $U(G F(2) S)$ is solvable and $S$ is a subdirect product of $G_{1}, \ldots, G_{m}$ with each $G_{i} \cong D_{3}$.

(ii) The group $S$ is a pairwise nondirect subdirect product of $G_{1}, \ldots, G_{m}$ with each $G_{i} \cong D_{3}$.

(iii) The group $S$ is an extension of an elementary abelian 3-group $N$ of order $3^{m}$ by an element a of order 2 which transforms each element of $N$ into its inverse.

Proof. (i) implies (ii). To see this let $F=G F(2), D_{3}=\langle a, b\rangle, a^{2}=b^{3}=1, b^{a}=b^{-1}$. If $S$ is not pairwise nondirect then $m>1$ and $U(F S)$ has a factor group of the form $U\left(F\left(D_{3} \times D_{3}\right)\right)$, which has in turn a nonsolvable subgroup $U\left(F\left(\langle b\rangle \times D_{3}\right)\right)$ according to Lemma 3.

To prove (ii) implies (iii) let $G_{i}=\left\langle a_{i}, b_{i}\right\rangle, a_{i}^{2}=b_{i}^{3}=1, b_{i}^{a_{i}}=b_{i}^{-1}$ and use induction. If $m=1$, then just let $S=D_{3}, N=\left\langle b_{1}\right\rangle$, and $a=a_{1}$. Assume for all $k<m$ that any pairwise nondirect subdirect product of $G_{1}, \ldots, G_{k}$ has the property of $S$ in (iii) and let $S$ be a pairwise nondirect subdirect product of $G_{1}, \ldots, G_{m}$. Then $S$ is a subdirect product of $S^{*}$ and $G_{m}$ where $S^{*}$ is a subdirect product of $G_{1}, \ldots, G_{m-1}$. Now $S^{*}$ is pairwise nondirect so $S^{*}=\left\langle N^{*}, a^{*}\right\rangle$ as in condition (iii). There are subgroups $N_{1} \Delta S^{*}$ and $N_{2} \Delta G_{m}$ and an isomorphism $\alpha: S^{*} / N_{1} \rightarrow G_{m} / N_{2}$ such that $\left(s^{*}, g_{m}\right) \in S$ if and only if $\alpha\left(s^{*} N_{1}\right)=g_{m} N_{2}$. If $\alpha\left(S^{*} / N_{1}\right)=G_{m} / N_{2}=1, \dot{S}=S^{*} \times G_{m}$, which means $S$ is not pairwise nondirect, a contradiction. If $\alpha\left(S^{*} / N_{1}\right) \cong D_{3}$, then $S=S^{*}$ and has the desired property. If $\alpha\left(S^{*} / N_{1}\right) \cong C_{2}$, then $N_{1}$ is of index 2, $N_{1}=N^{*}, N_{2}=\left\langle b_{m}\right\rangle$, and $S=\left(N^{*} \times\left\langle b_{m}\right\rangle\right) \cup\left(a^{*} N \times a_{m}\left\langle b_{m}\right\rangle\right)$. Let $N=N^{*} \times\left\langle b_{m}\right\rangle$ and $a=\left(a^{*}, a_{m}\right)$. Then $a \in S, a^{2}=1$ and $\left(n^{*}, b_{m}^{i}\right)^{a}=\left(n^{*}, b_{m}^{i}\right)^{-1}$ for every $n^{*}$ in $N^{*}$ and every $i$. Hence $S$ has the desired property. 
And finally we prove (iii) implies (i). Consider $S=\langle N, a\rangle$ as in (iii). Any subgroup of $N$ is normal in $S$ and $N=S^{\prime}$. Hence the absolutely irreducible linear representations of $S$ correspond one-to-one with those of $S / N \cong C_{2}$; at characteristic 2 there is only one such representation. The 2-regular classes of $S$ are $\{1\}$ and all pairs $\left\{n, n^{-1}\right\}$ for $n$ in $N$ since $n_{1}^{a}=n_{1}^{-1}$ but $n_{1}^{n_{2}}=n_{1}$ for $n_{1}, n_{2}$ in $N$; there are thus $\left(3^{m}-1\right) / 2$ 2-regular classes different from $\{1\}$. But this is just the number of subgroups of index 3 in $N$, which is the number of normal subgroups of index 6 in $N$. To see this, notice that the number of ordered bases for a fixed maximal $G F(3)$ subspace $N^{*}$ of $N$ times the number of complementary vectors for $N^{*}$ is just the number of ordered bases for $N$ such that all but the last vector form together a basis for $N^{*}$. This number, $\left(3^{n-1}-1\right) \cdots\left(3^{n-1}-3^{n-2}\right)\left(2 \cdot 3^{n-1}\right)$, divided into the number $\left(3^{n}-1\right) \cdots\left(3^{n}-3^{n-1}\right)$ of ordered bases for $N$, gives the number of subgroups of index 3 in $N$. Such subgroups do not contain $S^{\prime}$, so $S$ modulo any one of them is isomorphic to $D_{3}$. These $\left(3^{m}-1\right) / 2$ subgroups are kernels for nonlinear absolutely irreducible representations for $S$ by $G L(2,2)$-in fact, for all the nonlinear ones. By Theorem 6, $U(G F(2) S)$ is solvable. The fact that $S$ is a product of $m$ copies of $D_{3}$ follows by induction on $m$. The result is trivial for $m=1$. If $S=\langle N, a\rangle$ with $N$ of order $3^{m}$ then $N=N^{*} \times\left\langle b_{m}\right\rangle$ with $b_{m}$ of order 3. Let $S^{*}$ $=\left\langle N^{*}, a\right\rangle$; by the induction hypothesis this is a subdirect product of $m-1$ copies of $D_{3}$. Next $S^{*} / N^{*} \cong D_{3} /\left\langle b^{\prime}\right\rangle \cong C_{2}$ where $D_{3}=\left\langle a^{\prime}, b^{\prime}\right\rangle$. Defining

$$
S^{\prime}=\left(N^{*} \times\left\langle b^{\prime}\right\rangle\right) \cup\left(a N^{*} \times a^{\prime}\left\langle b^{\prime}\right\rangle\right),
$$

we have a subdirect product of $S^{*}$ and $D_{3}$, hence of $m$ copies of $D_{3}$. But $S^{\prime}=\left\langle N,\left(a, a^{\prime}\right)\right\rangle$ and $\left(n_{1}, n_{2}\right)^{\left(a, a^{\prime}\right)}=\left(n_{1}^{a}, n_{2}^{a^{\prime}}\right)=\left(n_{1}, n_{2}\right)^{-1}$ for $n_{1}$ in $N^{*}$ and $n_{2}$ in $\left\langle b^{\prime}\right\rangle$; hence, $S^{\prime} \cong S$.

THEOREM 10. Suppose $F$ is a field of characteristic 2 and $G$ is a finite group such that $G^{\prime}$ is not a 2-group. Then $U(F G)$ is solvable if and only if $F=G F(2)$ and $G^{*}=G / O_{2}(G)$ is a pairwise nondirect subdirect product of copies of $D_{3}$.

Proof. As before, let $\rho_{1}, \ldots, \rho_{s}$ be the absolutely irreducible representations of $G$ at characteristic 2 where $\rho_{1}, \ldots, \rho_{l}$ are the linear ones. If $\rho_{i}$ is linear, then $\rho_{i}(G)$ is a subgroup of nonzero elements of a field of characteristic 2 and so $\rho_{i}(G)$ is cyclic of odd order. If $l=s, G^{*}$, as a subdirect product of $\rho_{1}(G), \ldots, \rho_{s}(G)$, is abelian, contrary to assumption; hence $l<s$.

Suppose $U(F G)$ is solvable. Then $F=G F(2)$ and $\rho_{i}(G) \cong G L(2,2)$ for $i>l$ according to Theorem 6. Thus $G^{*}$ is a subdirect product of odd order cyclic groups $\rho_{1}(G), \ldots, p_{l}(G)$ and one or more copies $\rho_{l+1}(G), \ldots, \rho_{s}(G)$ of $D_{3}$. This makes $G^{*}$ a subdirect product of $A$ and $B$ for an odd order abelian $A$ and a subdirect product $B \neq 1$ of copies of $D_{3}$. There are subgroups $N_{1} \Delta A, N_{2} \Delta B$, and an isomorphism $A / N_{1} \cong B / N_{2}$. Since $A / N_{1}$ is of odd order, $A / N_{1} \cong B / N_{2}=1, G^{*} \cong A \times B$; so by Lemma 3 and the fact that $U\left(F G^{*}\right)$ is solvable, $A=1$ and $G^{*} \cong B$. Thus $U(F B)$ is solvable and $B$ is pairwise nondirect by Theorem 9 . 
Conversely, if $F=G F(2)$ and $G^{*}$ is such a product of copies of $D_{3}$, then by Theorem $9, U\left(F G^{*}\right)$ is solvable. Then by Theorem $6, U(F G)$ is solvable because representations of $G^{*}$ are essentially representations of $G$.

THEOREM 11. Suppose $F$ is a field of characteristic 3 and $G$ is a finite group such that $G^{\prime}$ is not a 3-group. Then $U(F G)$ is solvable if and only if $F=G F(3)$ and $G^{*}=G / O_{3}(G)$ is a 2-group with $e\left(G^{*}\right)=\left(\left|G^{*}\right|-\left[G^{*}: G^{* \prime}\right]\right) / 4$ normal subgroups of index 8 that do not contain $G^{* \prime}$.

Proof. Assume $U(F G)$ solvable. Then $F=G F(3)$ and $G^{*}$ is a subdirect product of $A$ and $B$ where $A$ is a subdirect product of cyclic groups $\rho_{1}(G), \ldots, \rho_{l}(G)$ of orders prime to 3 and $B$ is a subdirect product of $\rho_{l+1}(G), \ldots, \rho_{s}(G)$, each of which is a 2-group isomorphic to $Q$ or $D_{4}$ by Theorems 6, 7 and Lemma 2. Then $A=A_{1} \times A_{2}$ where $A_{1}$ is odd-order abelian and $A$ is an abelian 2-group. Hence $G^{*}$ is a subdirect product of $A_{1}$ and $C$ where $C$ is a subdirect product of $A_{2}$ and $B$ and is a 2-group. Since $\left(\left|A_{1}\right|,|C|\right)=1, G^{*} \cong A_{1} \times C$. Since $G^{*}$ is nonabelian, $C$ is too. By Lemma $3, A_{1}$ is an elementary abelian 2-group, so $A_{1}=1$ and $G^{*} \cong C$.

By Lemma 1, $G^{*}$ has $e\left(G^{*}\right)$ absolutely irreducible representations of degree 2 . The kernels of these representations must be the normal subgroups of index 8 in $G^{*}$ that fail to contain $G^{* \prime}$ since the images are of the form $Q$ or $D_{4}$. Consequently, $G^{*}$ has exactly $e\left(G^{*}\right)$ such subgroups.

Conversely, if $F=G F(3)$ and $G^{*}$ is a 2-group which has exactly $e\left(G^{*}\right)$ normal subgroups $N_{i}$ of index 8 that do not contain $G^{* \prime}$, then there is a different absolutely irreducible representation for each $N_{i}$ and each such representation is of degree 2 and definable over $G F(3)$. But from the equation

$$
\left|G^{*}\right|=\sum_{i=1}^{s} n_{i}^{2}=l+4(b-l)+\sum_{i=b+1}^{s} n_{i}^{2},
$$

where $n_{i}>2$ if and only if $i>b$ and the $n_{i}$ 's are the degrees of the absolutely irreducible representations of $G^{*}$, we see that $\sum_{i=b+1}^{s} n_{i}^{2}=0$ so $b=s$ and so by Theorem $6, U(F G)$ is solvable.

The last result can be refined by adding necessary conditions for $U\left(F G^{*}\right)$ to be solvable.

THEOREM 12. Suppose $U(F S)$ is solvable, $F=G F(3)$, and $S$ is a nonabelian 2group. Then $S$ is a subdirect product of copies of $C_{2}, C_{4}, D_{4}$, and $Q$.

Proof. With $m>1$ let $S$ be a subdirect product of $C_{2^{m}}=\langle c\rangle$ and $Q$. Then $S=\langle c\rangle \times Q$ or $S=\langle c\rangle * Q=\left\langle c^{2}\right\rangle \times\langle i\rangle \cup c\left\langle c^{2}\right\rangle \times j\langle i\rangle$ where $i$ and $j$ are noncommuting elements of $Q$ of order 4 . For $m \geqq 3$ there is an epimorphism $\alpha: C_{2^{m}} \rightarrow C_{8}$ with kernel $\alpha \leqq\left\langle c^{2}\right\rangle$. Hence there are epimorphisms $C_{2^{m}} \times Q \rightarrow C_{8} \times Q$ and $C_{2^{m}} * Q \rightarrow C_{8} * Q$. It suffices to show that for $m=3 U(F S)$ is not solvable. Let $m=3$. By Lemma $3,\langle c\rangle \times Q$ does not have a solvable unit group over $F$. Consider $S=\langle c\rangle * Q$, of order 32. Obviously, $S=\left\langle\left(c^{2}, 1\right),(1, i),(c, j)\right\rangle, S^{\prime}$ 
$=\langle(1,-1)\rangle$ of index 16, and $e(S)=4$. The subgroups $N_{1}=\left\langle\left(c^{2}, 1\right)\right\rangle$ and $N_{2}$ $=\left\langle\left(c^{2},-1\right)\right\rangle$ are normal, are of index 8 , and do not contain $S^{\prime}$. Suppose $N$ is another such group. Then $N \leqq\left\langle c^{2}\right\rangle \times\langle i\rangle$ since $c$ is of order 8 , and $N$ cannot contain $\left(c^{2}, 1\right),\left(c^{6}, 1\right),\left(c^{2},-1\right)$, or $\left(c^{6},-1\right)$ since any one of them generates $N_{1}$ or $N_{2}$. Also $(1,-1) \notin N$ since it generates $S^{\prime}$. If $g=\left(c^{2 q}, \pm i\right) \in N$, then $(1,-1)=g^{-1} g^{\left(c_{i j}\right)} \in N$. The only elements of $\left\langle c^{2}\right\rangle \times\langle i\rangle$ that $N$ could contain are $(1,1),\left(c^{4}, 1\right)$, and $\left(c^{4},-1\right)$, an absurdity. Hence $N_{1}$ and $N_{2}$ are the only possibilities and $U(F S)$ is not solvable. It follows that for $m \geqq 3$ the subdirect products $S$ of $C_{2^{m}}$ and $Q$ do not have solvable $U(F S)$.

Let $S$ be a subdirect product of $C_{2^{m}}=\langle c\rangle$ and $D_{4}=\langle a, b\rangle, a^{2}=b^{4}=1, b^{a}=b^{-1}$. Then, up to an isomorphism, $S=\langle c\rangle \times D_{4}, S=\langle c\rangle * D_{4}=\left\langle c^{2}\right\rangle \times\langle b\rangle \cup c\left\langle c^{2}\right\rangle \times a\langle b\rangle$, or $S=\langle c\rangle * * D_{4}=\left\langle c^{2}\right\rangle \times\left\langle a, b^{2}\right\rangle \cup c\left\langle c^{2}\right\rangle \times b\left\langle a, b^{2}\right\rangle$. Again it suffices to consider $m=3$. First, $U(F S)$ is not solvable if $S=\langle c\rangle \times D_{4}$. Consider secondly $S=\langle c\rangle * D_{4}$, of order 32. We have $S=\left\langle\left(c^{2}, 1\right),(1, b),(c, a)\right\rangle, S^{\prime}=\left\langle\left(1, b^{2}\right)\right\rangle$ of index 16 , and $e(S)=4$. The only two normal subgroups of index 8 not containing $S^{\prime}$ are $N_{1}=\left\langle\left(c^{2}, 1\right)\right\rangle$ and $N_{2}=\left\langle\left(c^{2}, b^{2}\right)\right\rangle$. Hence $U(F S)$ is not solvable. Thirdly, the group $S=\langle c\rangle * * D_{4}$ has order 32 . Clearly we have $S=\left\langle\left(c^{2}, 1\right),(1, a),\left(1, b^{2}\right),(c, b)\right\rangle$, so $S^{\prime}=\left\langle\left(1, b^{2}\right)\right\rangle$ of index 16 , and $e(S)=4$. Again we have only $N_{1}=\left\langle\left(c^{2}, 1\right)\right\rangle$ and $N_{2}=\left\langle\left(c^{2}, b^{2}\right)\right\rangle$, so $U(F S)$ is not solvable. Thus for $m \geqq 3$ if $S$ is a subdirect product of $C_{2^{m}}$ and $D_{4}$ then $U(F S)$ is not solvable.

Finally, if $S$ is a 2-group with $U(F S)$ solvable, then $S$ is a subdirect product of cyclic 2-groups and copies of $Q$ and $D_{4}$. If $S$ is also nonabelian, then at least one copy $H$ of $Q$ or $D_{4}$ will be present. If $C_{2^{m}}$ is one of the cyclic groups, then some subdirect product of $C_{2^{m}}$ and $H$ is a homomorph of $S$. Hence $m<3$ for each $C_{2^{m}}$ in the factorization of $S$.

Corollary 1. If $S$ is a nonabelian 2-group with $U(G F(3) S)$ solvable, then $S$ has exponent $4, Z(S)$ has exponent $2, S / Z(S)$ has exponent 2 , and conjugate elements of $S$ are congruent modulo $Z(S)$.

Proof. A subdirect product of groups of exponents 2 or 4 must have exponent 2 or 4 . A group $S$ of exponent 2 would be abelian.

Consider $S \leqq T_{1} \times \cdots \times T_{n}$ with each $T_{i}$ isomorphic to $C_{2}, C_{4}, D_{4}$, or $Q$. Then if $h=\left(h_{1}, \ldots, h_{n}\right) \in Z(S)$, each $h_{\alpha}$ is central in $T_{\alpha}$. If $T_{\alpha}=C_{4}=\left\langle c_{\alpha}\right\rangle$ and $T_{\beta}=Q$ or $D_{4}$ (which happens for at least one $\beta$ ), then $h_{\beta}= \pm 1 \in Q$, or $h_{\beta}=b^{2} \in D_{4}$. Since $H$ $=\left\{\left(h_{\alpha}, h_{\beta}\right) \mid\left(h_{i}, \ldots, h_{n}\right) \in S\right\}$ has solvable $U(F H), h_{\alpha}=1$ or $c_{\alpha}^{2}$. Obviously, $h_{\alpha}^{2}=1$ and $h_{\beta}^{2}=1$. Therefore $Z(S)$ has exponent 2 .

If $T=Q$ or $D_{4}$, then $Z(T)$ has exponent 2 and $T / Z(T)$ has exponent 2. Let $g \in S$. Then $g^{2}=\left(g_{1}^{2}, \ldots, g_{n}^{2}\right)$, each $g_{\beta}^{2}$ with $T_{\beta}=Q$ or $D_{4}$ is central, the other factors $g_{\alpha}^{2}$ are central in the cyclic groups $T_{\alpha}$. Thus $g^{2} \in Z(S)$ and $S / Z(S)$ has exponent 2.

Since conjugate elements of $C_{2}, C_{4}, Q$, or $D_{4}$ are congruent modulo their respective centers, the same property is possessed by any subdirect product $S$ of such groups. 
COROLlary 2. If $S$ is a nonabelian 2-group and $U(G F(3) S)$ is solvable, then $S=E \times I$ where $E$ is elementary abelian and $I$ is an indecomposable nonabelian subdirect product of copies of $C_{4}, Q$, and $D_{4}$. Conversely, if $U(G F(3) I)$ is solvable, $I$ is a 2-group, and $E$ is an elementary abelian 2-group, then $U(G F(3)(E \times I))$ is solvable.

Proof. It is clear from Lemma 3 that $S=E \times I$ with $E$ elementary abelian and $I$ indecomposable and nonabelian. Now $I$ is a subdirect product of $T_{1}, \ldots, T_{m}$ where each $T_{\alpha}$ is of the form $C_{2}, C_{4}, Q$, or $D_{4}$. If $T_{\alpha} \cong C_{2}$, then $I$ is a subdirect product of $T_{\alpha}$ and a nonabelian $I_{1}$. But $I \nsubseteq T_{\alpha} \times I_{1}$, hence $I \cong I_{1}$ where $I_{1}$ is a subdirect product of $\left\{T_{\beta}\right\}_{\beta \neq \alpha}$. An inductive argument shows that all $T_{\alpha} \cong C_{2}$ can be ignored. The converse is immediate from Lemma 3.

The necessary conditions presented in Theorem 12 and its corollaries are not sufficient. The group

$$
S=\left\langle(a, 1),\left(b^{2}, 1\right),(1, b),(b, c)\right\rangle
$$

is a subdirect product of $D_{4}$ with $D_{4}$; but $U(G F(3) S)$ is not solvable.

THEOREM 13. If $G$ is finite and $F$ has characteristic $p \geqq 0$, then $U(F G)$ is nilpotent if and only if $G=A \times P$ for a p-group $P$ and an abelian group $A$.

Proof. If $G=A \times P, F G \cong F A \otimes F P \cong\left(\sum F_{i}\right) \otimes F P$ for fields $F_{i}$ over $F$. Thus $F G \cong \sum\left(F_{i} \otimes F P\right) \cong \sum F_{i} P$, and $U(F G) \cong{ }^{\times} \prod U\left(F_{i} P\right)$. But each

$$
U\left(F_{\imath} P\right) \cong F_{i}^{*} \times\left(1+\operatorname{rad} F_{i} P\right)
$$

[4], so that $U\left(F_{i} P\right)$ is nilpotent. Hence $U(F G)$ is nilpotent.

Conversely, if $U(F G)$ is nilpotent, then $G \leqq U(F G)$ is nilpotent, so that $G=P \times P^{*}$, where $P^{*}$ is a $p^{\prime}$-group. Now $U\left(F P^{*}\right)$ is nilpotent-hence, solvableso $U\left(F P^{*}\right) \cong{ }^{\times} \prod_{i} G L\left(n_{i}, P\right)$ where each $n_{i}=1$ or 2 . In fact, no $n_{i}=2$, for no $G L(2, p)$ is nilpotent. Hence $P^{*}$ is abelian.

Theorem 13 appeared in [2].

Lemma. Let $D$ be a division ring. Then $U(D)$ is supersolvable if and only if $D$ is finite.

Proof. If $D$ is finite, $U(D)$ is finite abelian. Conversely, if $U(D)$ is supersolvable then there exist $\alpha_{1}, \ldots, \alpha_{m}$ such that each $\delta \neq 0$ in $D$ has the form $\prod_{i=1}^{m} a_{i}^{e_{i}}$. If $\alpha_{\imath}$ has infinite order, $\alpha_{i}$ is transcendental over $Z_{p}$. But then $U\left(Z_{p}\left(\alpha_{i}\right)\right)$ is not finitely generated because there is an infinite set of unassociated primes in $Z_{p}\left[\alpha_{i}\right]$. Thus each $\alpha_{i}$ has finite order and $D$ is finite.

THEOREM 14. Let $A$ be a finite-dimensional F-algebra with 1 that is separable modulo its radical. Then $U(A)$ is supersolvable only if $F$ is finite and in the decomposition $A / \operatorname{rad} A \cong \sum M_{n_{i}}\left(D_{i}\right)$, each $n_{i}=1$ or 2 , each $D_{i}$ is a field, and if some $n_{i}=2$ then $D_{i}=F=G F(2)$. 
Proof. Suppose $U(A)$ supersolvable. Then $U(F) \leqq U(A)$ so $F$ is finite. Also $U(A)$ is solvable but $G L(2,3)$ is not supersolvable so the remaining conditions are necessary.

THEOREM 15. Let $A$ be as in Theorem 14. Then $U(A)$ is p-solvable if and only if $A$ is finite and $U(A)$ is solvable.

Proof. Let $\bar{p}$ be a prime and $A / \operatorname{rad} A \cong \sum M_{n_{i}}\left(D_{i}\right)$. Suppose $U(A)$ is $\bar{p}$-solvable, then $A$ is finite and each $D_{i}$ is a field $F_{i}$. Also $U(A)$ is $\bar{p}$-solvable if and only if each $G L\left(n_{i}, F_{i}\right)$ is since $U(A) / O_{p}(U(A)) \cong \prod G L\left(n_{i}, F_{i}\right)$. If $G L(m, K)$ is $p$-solvable, $K=G F(q)$, and $m>1$, then $\left(1+q+\cdots+q^{m}\right) q^{m-1}$ divides $|P S L(m, q)|$ and so $\operatorname{PSL}(m, q)$ is not simple, i.e., $q=2$ or 3 and $m=2$. Conversely, if $m=1$ or if $m=2$ and $q=2$ or 3 , then $G L(m, q)$ is $p$-solvable. The result now follows from Theorem 5 .

REMARKS. The first paragraph of the above proof of Theorem 13 is from the author's dissertation and provides a correction to [2]. Bhattacharya and Jain found a counterexample to the lemma of [2] and announced a correction (see Notices of the American Mathematical Society, April 1969). The finiteness part of Theorem 14 was obtained independently by Eldridge in the more general setting of Artinian rings (see Notices of the American Mathematical Society, August 1968).

\section{REFERENCES}

1. E. Artin, Geometric algebra, Interscience, New York, 1957. MR 18, 553.

2. J. M. Bateman and D. B. Coleman, Group algebras with nilpotent unit groups, Proc. Amer. Math. Soc. 19 (1968), 448-449. MR 36 \#5238.

3. M. Burrow, Representation theory of finite groups, Academic Press, New York, 1965. MR 38 \#250.

4. D. B. Coleman, On the modular group ring of a p-group, Proc. Amer. Math. Soc. 15 (1964), 511-514. MR 29 \#2306.

5. C. W. Curtis and I. Reiner, Representation theory of finite groups and associative algebras, Pure and Appl. Math., vol. 11, Wiley, New York, 1962. MR 26 \#2519.

6. W. E. Deskins, Finite abelian groups with isomorphic group algebras, Duke Math. J. 23 (1956), 35-40. MR 17, 1052.

7. M. Hall, Jr., The theory of groups, Macmillan, New York, 1959. MR 21 \#1996.

8. L. K. Hua, On the multiplicative groups of a field, Acad. Sinica Sci. Record 3 (1950), 1-6. MR 12, 584.

9. J. Rotman, The theory of groups. An introduction, Allyn and Bacon, Boston, Mass., 1965. MR 34 \#4338.

Michigan State University,

East Lansing, Michigan 48823 